\title{
Universal mechanism of tetrahedral metal cluster formation in structures with breathing pyrochlore sublattices
}

\author{
M. V. Talanov ${ }^{1}$, V.M. Talanov ${ }^{2}$ \\ ${ }^{1}$ Research Institute of Physics, Southern Federal University, Rostov-on-Don, Russia \\ ${ }^{2}$ South-Russian State Polytechnical University, Novocherkassk, Russia \\ tmikle-man@mail.ru, valtalanov@mail.ru
}

PACS 61.50.Ks; 36.40.-c

DOI 10.17586/2220-8054-2017-8-5-677-687

\begin{abstract}
A universal mechanism of tetrahedral metal cluster formation in crystal with geometrically frustrated pyrochlore sublattices is proposed. It has been shown that the critical irreducible representation $\tau$, which generated the formation of metal clusters in non-centrosymmetrical F $\overline{4} 3 \mathrm{~m}$-phases from high symmetry phases with $\mathrm{Fd} \overline{3} \mathrm{~m}$ space group, is a one dimensional irreducible representation $\kappa_{11}\left(\tau_{4}\left(\mathrm{~A}_{2 u}\right)\right.$ ) (in Kovalev notation). The structural theory of metal cluster formation based on group theoretical calculations was published earlier for the case of A-ordered spinel. In this work, the theory is generalized in the case of any high symmetry Fd $\overline{3} \mathrm{~m}$ structures that include pyrochlore sublattices. We presented a brief review of such structures and mechanisms of the tetrahedral metal cluster formation. The existence of so called "breathing" pyrochlore sublattices in ordered phases is predicted theoretically. The groups of atoms, between which bond clusters, are found. These groups of atoms define electron correlation effects. Examples of tetrahedral metal cluster formation in ordered spinels, ordered lacunar spinels, ordered Laves phases $\left(\mathrm{MgCu}_{4} \mathrm{Sn}\right.$ structural type) and ordered pyrochlore are considered. The theoretical results are confirmed by the known experimental facts.
\end{abstract}

Keywords: tetrahedral clusters, ordered spinel, ordered Laves phases, ordered pyrochlore, geometrical frustration, breathing pyrochlore sublattices.

Received: 10 August 2017

Revised: 1 September 2017

\section{Introduction}

Geometric frustration is an old topic of physics and solid state chemistry [1-4]. L. Pauling, using the example of crystalline ice, has shown the existence of different orientations of water molecules, which do not lead to a change in the energy of the system $[5,6]$.

The paradigm of geometric frustration is used in different scientific fields: for the description of the nontrivial packing of polyhedra in three-dimensional space in systems such as quasi-crystals; for amorphous metals and nematic disclination networks in densely packed 3D colloidal lattices; in the theory of magnetism when the arrangement of spins on a lattice does not satisfy all interactions at the same time; for the study of the orientation order in the proton configurations in ordinary hexagonal ice and for spins in magnetic molecules and artificial magnetic architectures.

The term "geometrical frustration" describes the structures with local order generated by the lattice geometry. Frustration arises when the geometry of any system allows for a set of degenerate ground states. Such highly degenerate systems are extremely sensitive to thermal and quantum fluctuations, and thereby intriguing classical and quantum ground states may emerge via "order by disorder".

The important physical properties of many inorganic crystals are connected with a structural feature - a threedimensional network of the tetrahedra formed by cations. A network of these tetrahedra is called a pyrochlore sublattice. Some such pyrochlore sublattices are in the following: spinels $\mathrm{AB}_{2} \mathrm{X}_{4}$ (where the $\mathrm{B}$ site displays a pyrochlore sublattice); pyrochlores $\mathrm{A}_{2} \mathrm{~B}_{2} \mathrm{O}_{7}$ (where both $\mathrm{A}$ and $\mathrm{B}$ sites form corner-sharing tetrahedra): and Laves phases (C15) $\mathrm{AB}_{2}$ (where B-metal atoms form a pyrochlore sublattice). There are hundreds of compounds crystallizing in these structural types. The geometrical frustration of spins results in the formation of exotic electronic and structural state in these substances. One of these states is spin liquid.

P. W. Anderson was the first to show that B-sublattice (pyrochlore sublattice) in spinel structure is geometrically frustrated and causes the unusual physical properties of this class of materials [4].

In this paper, we theoretically establish the universal structural mechanism for the formation of metal clusters namely metal tetrahedra in inorganic crystals with $F \overline{4} 3 \mathrm{~m}$ space group. This short paper is written from a solid state chemist's point of view, with an emphasis on crystalline materials and structural chemistry aspects. 


\section{Symmetry of order parameter of $\mathbf{F d} \overline{3} m \leftrightarrow F \overline{4} 3 m$ phase transition}

The crystal structures of spinels, pyrochlores and Laves phases (C15) are characterized by the space group $\mathrm{Fd} \overline{3} \mathrm{~m}$. The subject of our investigation is the ordered phases of these structural types with the $\mathrm{F} \overline{4} 3 \mathrm{~m}$ space group. By using the concept of one critical irreducible representation (irrep) we will derive theoretically the structures of low-symmetry ordered cubic $\mathrm{F} \overline{4} 3 \mathrm{~m}$-phases from structures of high-symmetry disordered $\mathrm{Fd} \overline{3} \mathrm{~m}$-phases. The structure of low-symmetry F $\overline{4} 3 \mathrm{~m}$-phase is determined by the ordering of atoms and their displacements in the initial (parent) structure. The structural mechanism of $\mathrm{F} \overline{4} 3 \mathrm{~m}$-phase formation is generated by critical and noncritical (improper) irreps of $\mathrm{Fd} \overline{3} \mathrm{~m}$ space group.

The phases with $\mathrm{F} \overline{4} 3 \mathrm{~m}$ symmetry can be generated by one-dimensional irrep $\kappa_{11}\left(\tau_{4}\left(\mathrm{~A}_{2 u}\right)\right)$ and can also be generated by two four-dimensional irreps $\kappa_{9}\left(\tau_{1}\right)$ and $\kappa_{9}\left(\tau_{4}\right)$ [7-10]. Below, we will only discuss the phase induced by the irrep $\kappa_{11}\left(\tau_{4}\right)$ of group $\mathrm{Fd} \overline{3} \mathrm{~m}$. The expression $\kappa_{j}\left(\tau_{i}\right)$ means the star of wave vectors $\kappa_{j}$, where $i$ is number of corresponding irrep $\tau$ for given star $j$ [7]. Critical irrep $\kappa_{11}\left(\tau_{4}\right)$ determines the symmetry and structure of low-symmetry phase near point of phase transition.

The Wyckoff positions splitting takes place in the low-symmetry phase as a result of phase transition. To find the splitting of Wyckoff positions in the structure of the highly-symmetric phase it is necessary to analyze the composition of permutation and mechanical representations of high-symmetry structure. We have found that the critical one dimensional irrep $\kappa_{11}\left(\tau_{4}\right)$ enters into the mechanical representation on 16c, 16d, 32e, 48f Wyckoff positions and enters into the permutation representation on $8 \mathrm{a}, 8 \mathrm{~b}, 32 \mathrm{e}, 48 \mathrm{f}$ Wyckoff positions [11-15].

Therefore the low-symmetry F $\overline{4} 3 \mathrm{~m}$-phases formation is accompanied by simultaneous displacements of atoms spaced on 16c, 16d, 32e, 48f Wyckoff positions as well as by ordering of atoms spaced on 8a, 8b, 32e, 48f Wyckoff positions.

\section{3. $\mathbf{B}_{4}$-clusters in A-ordered spinel structures}

The structural formula of cubic centrosymmetric Fd 3 m-spinel $\mathrm{AB}_{2} \mathrm{X}_{4}$ is $(\mathrm{A})^{8 a}\left[\mathrm{~B}_{2}\right]^{16 d} \mathrm{X}_{4}^{32 e}$. The formation of a low-symmetry non-centrosymmetric $\mathrm{F} \overline{4} 3 \mathrm{~m}$-phase is accompanied by ordering of tetrahedral cations (1:1 order type) and anions (1:1 order type), and also by displacements of octahedral B-cations and anions. The calculated structure of the ordered $F \overline{4} 3 \mathrm{~m}$-phase is shown in Fig. 1. The results of the calculations demonstrate that the tetrahedral cations in the ordered phase occupy nonvariant $4 \mathrm{a}$ and $4 \mathrm{c}$ Wyckoff positions (site symmetry $\overline{4} 3 \mathrm{~m}$ ). The octahedral cations are displaced by equal distances along the four threefold axes and occupy monovariant Wyckoff positions 16e with local symmetry $3 \mathrm{~m}$ in the ordered structure (Figs. 1,2). The free parameter $x_{1}$ is approximately equal to 0.675. It is interesting that the B-cations and anions form specific clusters (Fig. 2(a, c, e)). The anionic lattice is separated into two sublattices in the ordered phase. The anions occupy the16c Wyckoff positions (site symmetry $3 \mathrm{~m})$. The anions' arrangement in each sublattice is described by the free parameters $x_{2} \approx 0.875$ and $x_{3} \approx 0.375$. The anions are displaced along the diagonals of the octants in the [111]-directions (Fig. 2a). It has been stated by calculation that general structural formula of the A-ordered spinel is $\mathrm{A}^{\prime 4 a} \mathrm{~A}^{4 c} \mathrm{~B}_{4}^{16 e} \mathrm{X}_{4}^{\prime 16 e} \mathrm{X}_{4}^{16 e}$ (Fig. 3) [16].

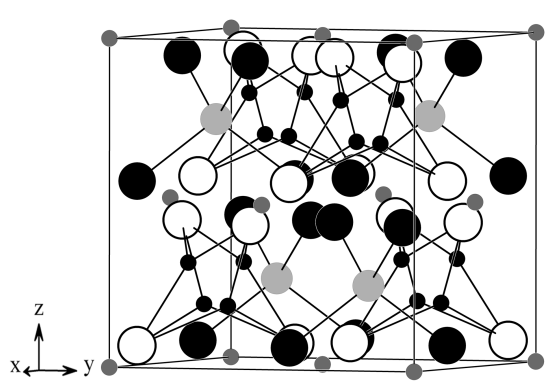

a

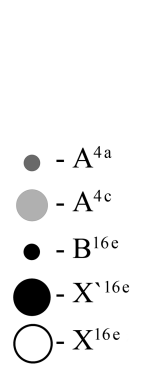

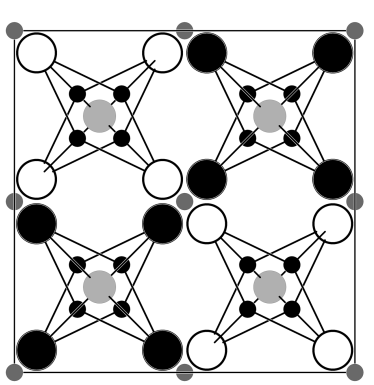

b

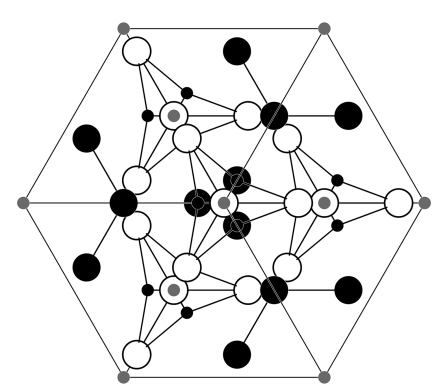

c

FIG. 1. Calculated structure of an A-ordered spinel (space group F̄̄3m). Atom presentation of the structure (a) and projections along (001) (b) and along (111) (c) of the ordered spinel structure

Features of the Fi্3m-phase structure are discussed in $[14,16]$. The metal clusters are the most interesting feature of this structure. Two neighboring groups of octahedral cations and anions form the expanded (Fig. 2(c, d)) and contracted (Fig. 2(b,e)) regular tetrahedra. Net of such alternative tetrahedra (expanded and contracted) forms the unusual pyrochlore sublattice with two different B-B distances [16] (Fig. 3, Table 1). In the work [17] this was termed "breathing" pyrochlore sublattice. Also it is interesting that the structural feature of ordered phase is "breathing" ring of tetrahedra (Fig. 4). 


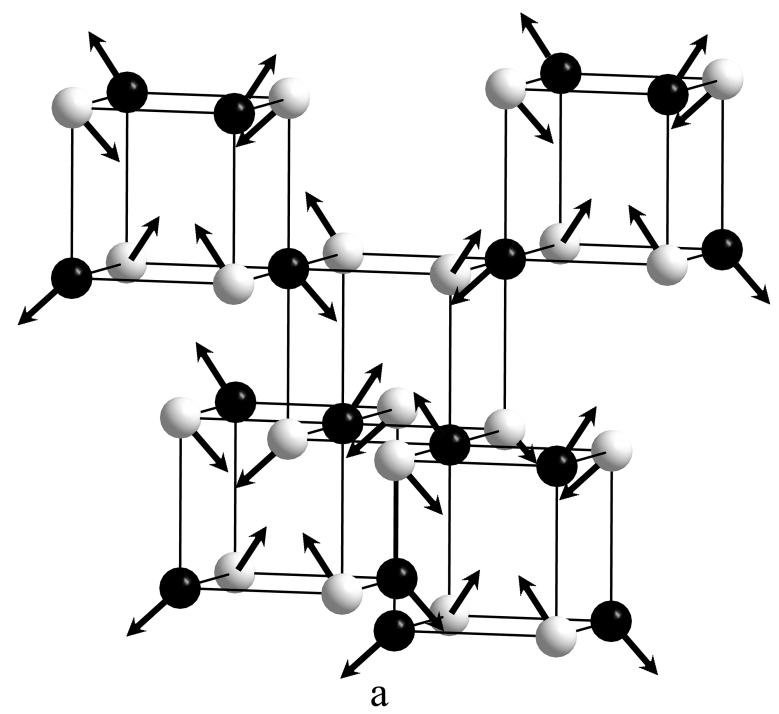

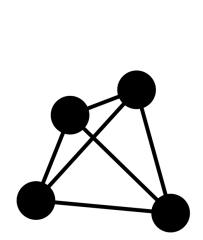

b

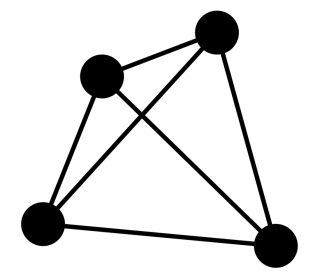

d

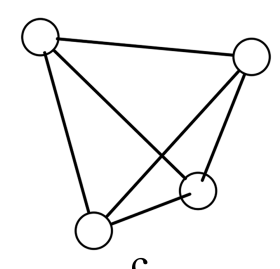

C

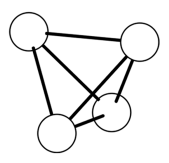

e

FIG. 2. Structural mechanism of atom cluster formation. Displacements of octahedral cations and anions in the adjacent octants of the spinel structure (a); compressed cluster $\mathrm{B}_{4}^{16 e}(\mathrm{~b})$, expanded cluster $\mathrm{X}_{4}^{16 e}(\mathrm{c})$, expanded cluster $\mathrm{B}_{4}^{16 e}(\mathrm{~d})$ and compressed cluster $\mathrm{X}_{4}^{16 e}(\mathrm{e})$

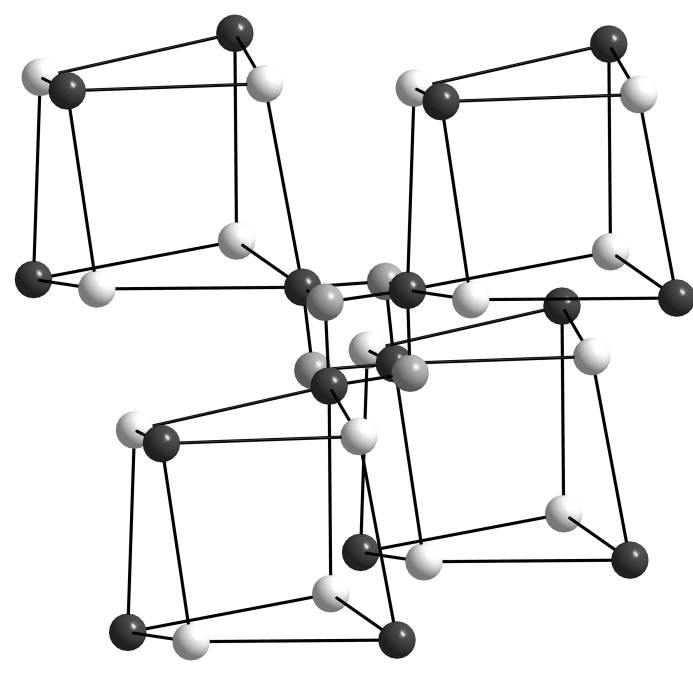

a

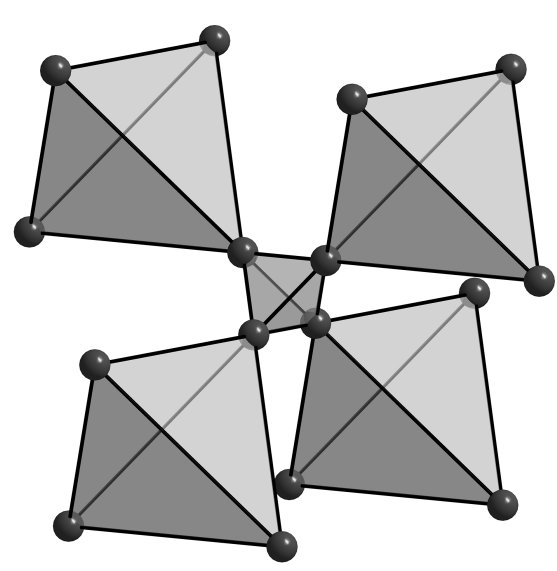

b

FIG. 3. Part of the crystal structure of spinel type with space group $F \overline{4} 3 \mathrm{~m}$ (a); expansion and contraction of the $\mathrm{B}_{4}$ tetrahedra are overdrawn (b). The designation of atoms is the same as in Fig. 1

The expanded and contracted tetrahedra of B-cations are considered as a metal cluster. Contracted tetrahedra have the linear size $\left(2^{1 / 2} / 4\right) a$ and are located in the distance $\left(6^{1 / 2} / 4\right) a$, where $a$ is the parameter of a cubic elementary cell of a spinel structure.

The chemical bonding between metal tetrahedra is realized with the help of bridge from two atoms $\mathrm{X}^{\prime 16 c}$ (Fig. 5). This bond influences the correlation of electron movement and the participant in the hopping mechanism of electrical conductivity for A-ordered spinels.

Metal tetrahedra form hyper-tetrahedra (Fig. 6). The hyper-tetrahedra are formed by 4 B-tetrahedra. The hyper-tetrahedra formed by B-cations may participate in the formation of magnetic properties of the ordered phase.

Metal clusters were experimentally observed in ordered spinels $\mathrm{LiXY}_{4} \mathrm{O}_{8}(\mathrm{X}=\mathrm{Ga}, \mathrm{Fe}, \mathrm{In} ; \mathrm{Y}=\mathrm{Cr}, \mathrm{Rh})$, $\mathrm{Cr}_{4} \mathrm{GaLiO}_{8}, \mathrm{Cr}_{4} \mathrm{InLiO}_{8}, \mathrm{Rh}_{4} \mathrm{InLiO}_{8}, \mathrm{Ag}_{0.5} \mathrm{In}_{0.5} \mathrm{Cr}_{2} \mathrm{~S}_{4}$ and $\mathrm{Cu}_{0.5} \mathrm{In}_{0.5} \mathrm{Cr}_{2} \mathrm{~S}_{4}$ [18-20] and their existence, apparently, causes unusual magnetic properties of these compounds. If such metal clusters have a magnetic moment, then it 
TABLE 1. Interatomic distances in the ordered structure of the spinel $\mathrm{A}_{1 / 2} \mathrm{~A}_{1 / 2}^{\prime} \mathrm{B}_{2} \mathrm{X}_{4}$

\begin{tabular}{|c|c|c|}
\hline \multirow[t]{2}{*}{ Atom pair } & \multicolumn{2}{|l|}{ Distances } \\
\hline & $\mathrm{F} \overline{4} 3 \mathrm{~m}$ from $\mathrm{Fd} 3 \mathrm{~m}$ & $\mathrm{~F} \overline{4} 3 \mathrm{~m}$ \\
\hline$A-X$ & $3^{1 / 2}(1 / 8+\delta-d)$ & $3^{1 / 2}\left(1-x_{2}\right)$ \\
\hline$A^{\prime}-X$ & $3^{1 / 2}(1 / 8+\delta-d)$ & $3^{1 / 2}\left(x_{2}-1 / 4\right)$ \\
\hline$A-X$ & {$\left[19 / 64+3(\delta-d)^{2}+5 / 4(d-\delta)\right]^{1 / 2}$} & {$\left[3 / 2+3 x_{2}^{2}\right]^{1 / 2}$} \\
\hline$A^{\prime}-X$ & {$\left[11 / 64+3(\delta-d)^{2}+1 / 4(\delta-d)\right]^{1 / 2}$} & {$\left[11 / 16-(3 / 2) x_{2}+3 x_{2}^{2}\right]^{1 / 2}$} \\
\hline $\mathrm{B}-\mathrm{X}$ & {$\left[(1 / 4-\delta+d-b)^{2}+2(d+b-\delta)^{2}\right]^{1 / 2}$} & {$\left[\left(x_{2}-x\right)^{2}+2\left(x_{2}+x-1 / 2\right)^{2}\right]^{1 / 2}$} \\
\hline $\mathrm{B}-\mathrm{X}$ & $3^{1 / 2}(1 / 4+d-\delta-b)$ & $3^{1 / 2}\left(x_{2}-x\right)$ \\
\hline $\mathrm{B}-\mathrm{X}$ & {$\left[2(3 / 8+\delta+d-b)^{2}+(-1 / 4+\delta+d-b)^{2}\right]^{1 / 2}$} & {$\left[2\left(x-x_{1}-1 / 2\right)^{2}+\left(x-x_{1}\right)^{2}\right]^{1 / 2}$} \\
\hline$A-A ; A^{\prime}-A^{\prime}$ & $(1 / 2) 2^{1 / 2}$ & $(1 / 2) 2^{1 / 2}$ \\
\hline $\mathrm{A}-\mathrm{A}^{\prime}$ & $(1 / 4) 3^{1 / 2}$ & $(1 / 4) 3^{1 / 2}$ \\
\hline $\mathrm{B}-\mathrm{B}$ & $2^{3 / 2}(1 / 8-b)$ & $2^{3 / 2}(3 / 4-x)$ \\
\hline $\mathrm{B}-\mathrm{B}$ & $2^{3 / 2}(1 / 8+b)$ & $2^{1 / 2}(2 x-1)$ \\
\hline$X-X$ & $2^{3 / 2}(1 / 8-\delta+x)$ & $2^{1 / 2}\left(2 x_{2}-1 / 2\right)$ \\
\hline
\end{tabular}

Note: $x=5 / 8+b ; x_{1}=u+d ; x_{2}=1 / 4-u+d$, where $b, d$-displacements of B-cations and X-anions, $u=3 / 8+\delta-$ anion parameter (free parameter).

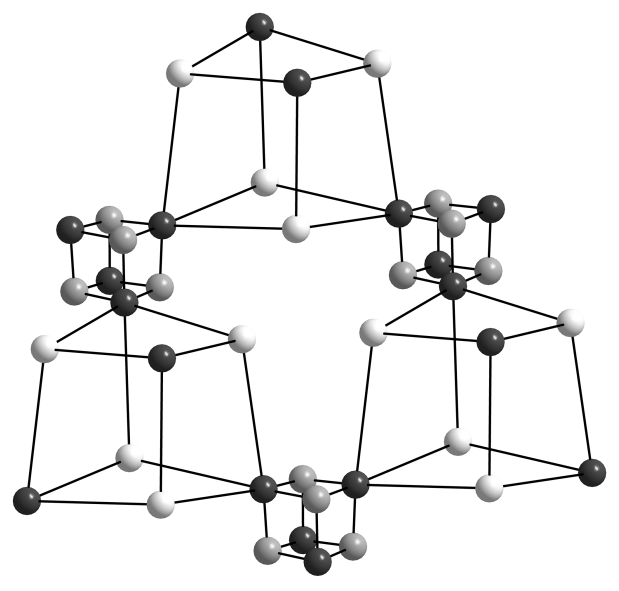

a

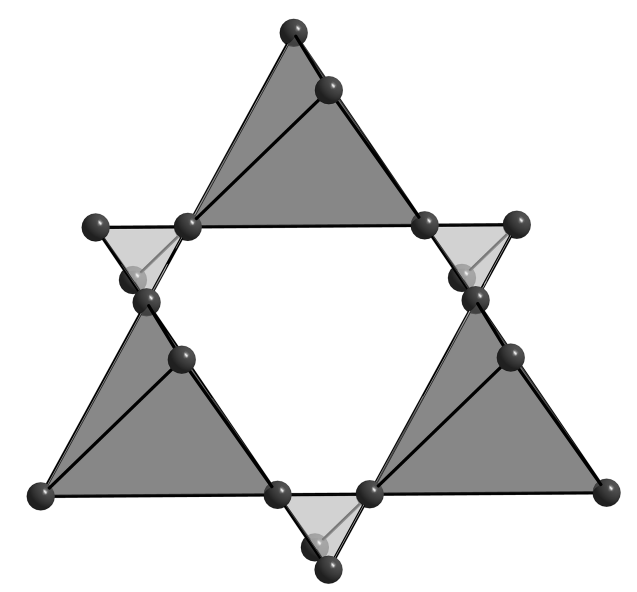

b

FIG. 4. B-cations in ordered spinel form "breathing" ring of tetrahedra. The designation of atoms is the same as in Fig. 1

is possible to expect that distribution of the magnetic moments on space of a crystal should be chaotic. In the results, the crystal which does not have a long ferromagnetic order, but has clusters with a certain degree of the local magnetic order, is formed. Such magnetic state is named spin glass. Thus, structural ordering of cations in spinel tetrahedral sites naturally causes an opportunity for the existence of a magnetic spin-glass state. In the case of magnetic interaction of metal tetrahedra unusual magnetic states of ordered spinels can be observed.

\section{4. $\mathbf{B}_{4}$-clusters in some lacunar ordered spinel structures}

The structure of some lacunar phases can be considered as a particular case of ordered spinel structures. The structure of these phases can be represented as a structure of defect spinels with the 1:1 ordering of cations in 

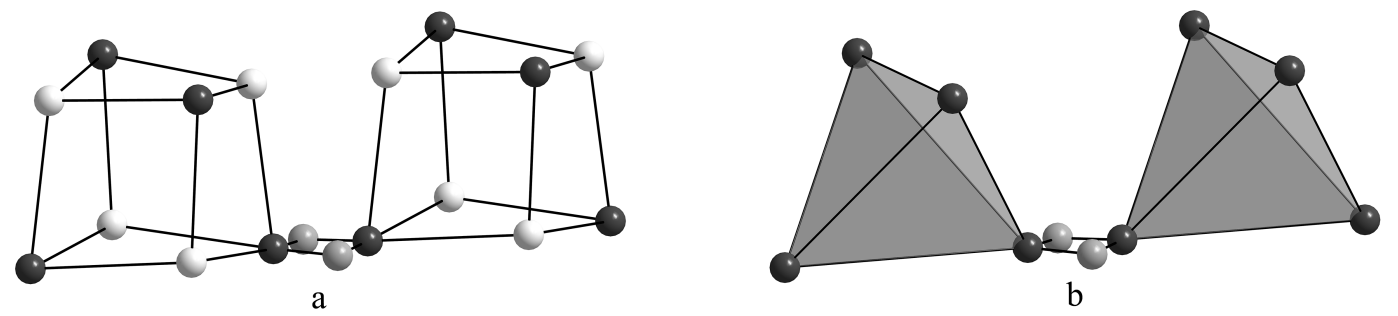

FIG. 5. The chemical bridge between two metal tetrahedra

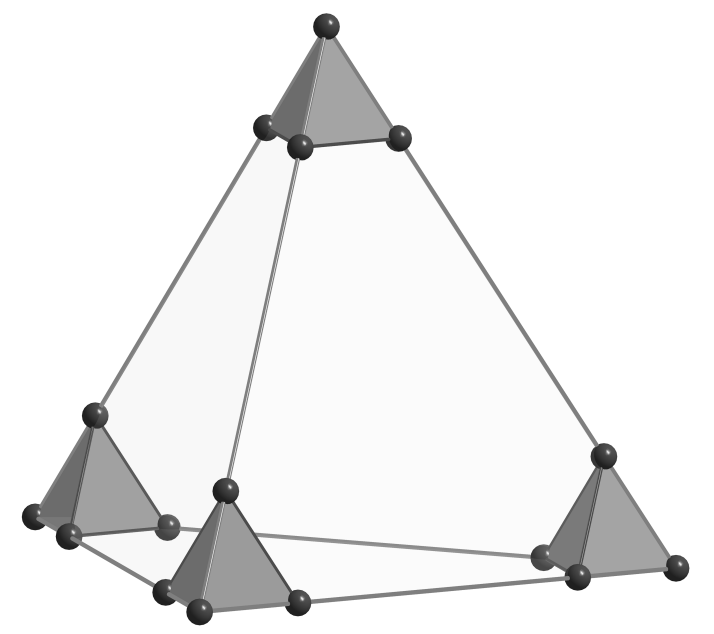

FIG. 6. The hyper-tetrahedra formed by B-catons

TABLE 2. The distribution of atoms in Wyckoff positions of some lacunar phases

\begin{tabular}{|c|c|c|c|c|c|c|c|}
\hline \multirow{2}{*}{$\begin{array}{c}\text { Examples of the } \\
\text { chemical compounds }\end{array}$} & \multirow{2}{*}{$\begin{array}{c}\text { Structural formula } \\
\end{array}$} & \multicolumn{5}{|c|}{ Wyckoff positions of $\mathrm{F} \overline{4} 3 \mathrm{~m}-\mathrm{phase}$} & \multirow{2}{*}{ References } \\
\cline { 3 - 8 } & & $\mathrm{A}^{\prime 4(a)}$ & $\mathrm{A}^{4(c)}$ & $\mathrm{B}_{4}^{16(e)}$ & $\mathrm{X}_{4}^{16(e)}$ & $\mathrm{X}_{4}^{\prime 16(e)}$ & \\
\hline $\mathrm{Re}_{4} \mathrm{~S}_{4} \mathrm{Te}_{4}$ & $\mathrm{~B}_{4}^{16 e} \mathrm{X}_{4}^{\prime 16 e} \mathrm{X}_{4}^{16 e}$ & $\square$ & $\square$ & $\mathrm{Re}_{4}$ & $\mathrm{~S}_{4}$ & $\mathrm{Te}_{4}$ & {$[21,22]$} \\
\hline $\mathrm{Ga}_{1.33} \mathrm{Cr}_{4} \mathrm{~S}_{8}$ & $\mathrm{~A}^{\prime 4 a} \mathrm{~A}^{4 c} \mathrm{~B}_{4}^{16 e} \mathrm{X}_{4}^{\prime 16 e} \mathrm{X}_{4}^{16 e}$ & $\mathrm{Ga}_{1.33}+\square$ & $\mathrm{Cr}_{4}$ & $\mathrm{~S}_{4}$ & $\mathrm{~S}_{4}$ & {$[23-25]$} \\
\hline $\mathrm{GaMo}_{4} \mathrm{O}_{8}$ & $\mathrm{~A}^{4 c} \mathrm{~B}_{4}^{16 e} \mathrm{X}_{4}^{\prime 16 e} \mathrm{X}_{4}^{16 e}$ & $\square$ & $\mathrm{Ga}$ & $\mathrm{Mo}_{4}$ & $\mathrm{O}_{4}$ & $\mathrm{O}_{4}$ & {$[26,27]$} \\
\hline $\mathrm{Re}_{4} \mathrm{As}_{6} \mathrm{~S}_{3}$ & $\mathrm{~A}^{4 c} \mathrm{~B}_{4}^{16 e} \mathrm{X}_{4}^{\prime 16} \mathrm{X}_{4}^{16 e}$ & $\square$ & $\mathrm{As}$ & $\mathrm{Re}_{4}$ & $\mathrm{As}_{4}$ & $\mathrm{AsS}_{3}$ & {$[28]$} \\
\hline
\end{tabular}

tetrahedral sites. Therefore, the lacunar structures and their properties are inherently associated with the structure of A-ordered spinels. All varieties of possible compositions of lacunar phases are theoretically deduced from the structural formula of A-ordered defect spinel with space group F $\overline{4} 3 \mathrm{~m}$ (Table 2). Lacunar phases of different types are obtained depending on the types of atoms that are absent in the structural formula of the A-ordered spinel. For example, Table 2 presents the experimentally revealed distributions of atoms on Wyckoff positions for some lacunar phases as particular cases of defect A-ordered spinel [21,22]. Defects in the table are designed by a symbol $\square$.

The lacunar structures "inherits" from the structure of the ordered spinel four types clusters, which have been established as a result of theoretical research of the structural mechanism of the spinel formation with the type 1:1 order in 8a and 32e Wyckoff positions (Section 3). There are two types of metal clusters - extended and compressed metal tetrahedra $\mathrm{B}_{4}^{16(e)}$ and two types of extended and compressed anion clusters $\mathrm{X}_{4}^{16(e)}$ and $\mathrm{X}_{4}^{\prime 16(e)}$. We believe that the formation of these clusters is the main reason of the unusual physical properties of these phases. There are many experimental examples which confirm with the considered concept of the universal mechanism of cluster formation [21-28]. 
The $\mathrm{Re}_{4}$ clusters in the $\mathrm{Re}_{4} \mathrm{Te}_{4} S_{4}$ (Fig. 7). The arrangement of the $\mathrm{ReS}_{3} \mathrm{Te}_{3}$ octahedra is the same as that in the $\mathrm{BX}_{6}$ octahedra in the spinel structure $\mathrm{AB}_{2} \mathrm{X}_{4}$, where $\mathrm{A}$ is a tetrahedrally coordinated metal atom and $\mathrm{B}$ is an octahedrally coordinated metal atom. According to our concept the $\mathrm{B}$ atom is at the centre of the $\mathrm{BX}_{6}$ octahedron in the spinel structure, but the Re atom is shifted from the centre of the $\mathrm{S}_{3} \mathrm{Te}_{3}$ octahedron to form $\mathrm{Re}_{4}$ cluster. The sites which are occupied by $A$ atoms in the spinel structure are empty in $\operatorname{Re}_{4} \mathrm{Te}_{4} \mathrm{~S}_{4}$ structure (Table 1, Fig. 5). In this structure $\mathrm{Re}_{4} \mathrm{~S}_{4}$ moieties are linked by bridging Te-groups. The part of rhenium atoms of this cluster may be replaced by molybdenum atoms, which lead to the formation of $\operatorname{Re}_{4-x} \mathrm{Mo}_{x}$ heterometallic cluster in $\mathrm{Re}_{4-x} \mathrm{Mo}_{x} \mathrm{~S}_{4} \mathrm{Te}_{4}(0 \leq x \leq 1.25)$ structure $[22,29]$.

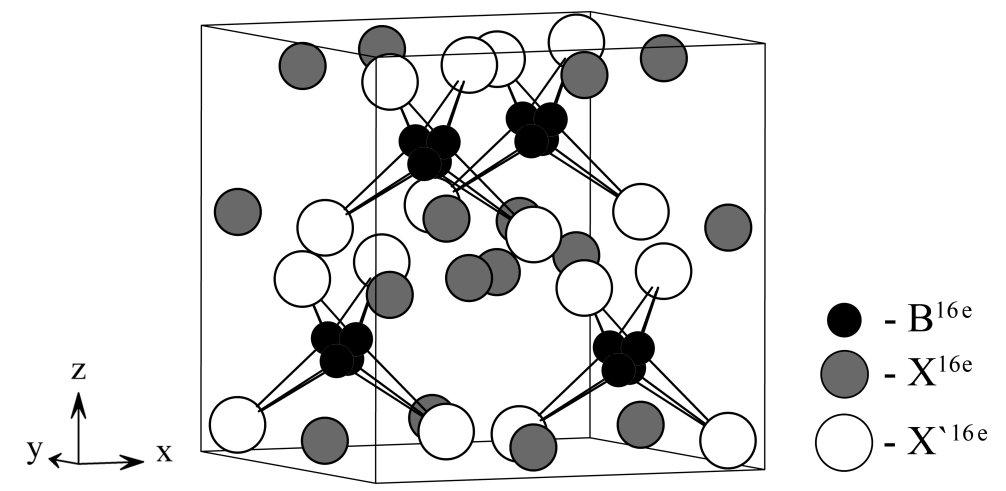

FIG. 7. The calculated structural motif of $\mathrm{Re}_{4} \mathrm{~S}_{4} \mathrm{Te}_{4}$ structure

The structure of the $\mathrm{Re}_{4} \mathrm{~S}_{4} \mathrm{Te}_{4}$ has been determined using X-ray diffraction [21,22]. The compound contains tetrahedral $\mathrm{Re}_{4}$ clusters with the $\mathrm{Re}-\mathrm{Re}$ distance of $2.785 \AA$ [21]. The distance between the $\operatorname{Re}_{4}$ clusters is equal to $4.345 \AA$ [30]. The geometry and arrangement of these $\operatorname{Re}_{4}$ clusters corresponds exactly to the $\mathrm{B}_{4}$-clusters arrangement on Figs. 1, 2. By introducing halogens into such rhenium-chalcogenide systems, related compounds, such as $\mathrm{Re}_{4} \mathrm{Te}_{4}\left(\mathrm{TeBr}_{2}\right)_{4} \mathrm{Br}_{8}$ [31] are obtained. It is interesting to note that the structure of $\mathrm{Re}_{4} \mathrm{Te}_{4} \mathrm{~S}_{4}$ is isotypic with those of the chalcogenohalides of niobium and molybdenum, $M_{4} X_{4} X^{\prime}{ }_{4}\left(M=N b, M o ; X=S, S e ; X^{\prime}=C 1\right.$, $\mathrm{Br}, \mathrm{I})[21,32,33]$.

The $\mathrm{Re}_{4}$ clusters in the $\operatorname{Re}_{4} A s_{6} S_{3}$ structure [28]. The $\mathrm{Re}_{4} \mathrm{As}_{6} \mathrm{~S}_{3}$ structure is isostructural to $\mathrm{GaMo}_{4} \mathrm{~S}_{8}$ and may be written as $\mathrm{AsRe}_{4}\left(\mathrm{As}_{0.25} \mathrm{~S}_{0.75}\right)_{4} \mathrm{As}_{4}$ according to the family of compounds $\mathrm{AB}_{4} \mathrm{X}_{4} \mathrm{Y}_{4}$. Anions in $32 \mathrm{e}$ Wyckoff position spinel splits into two 16-fold positions in the $\mathrm{F} \overline{4} 3 \mathrm{~m}$-phase. These two positions are occupied by the same atoms in the thio-spinel $\mathrm{AB}_{2} \mathrm{~S}_{4}$; but they may also contain chemically different atoms in case of more general family of compounds with structural formula $\mathrm{AA}^{\prime} \mathrm{B}_{4} \mathrm{X}_{4} \mathrm{X}^{\prime}{ }_{4}$. In $\mathrm{Re}_{4} \mathrm{As}_{6} \mathrm{~S}_{3}$, one of these positions is occupied entirely by arsenic atoms (IV) whereas the other contains a random mixture of sulfur and arsenic atoms (II) in a $3: 1$ ratio. As a consequence, the simple close packed anion lattice in the thio-spinels now consists of two distinct sublattices, producing a distorted close packed anion lattice (Fig. 8).

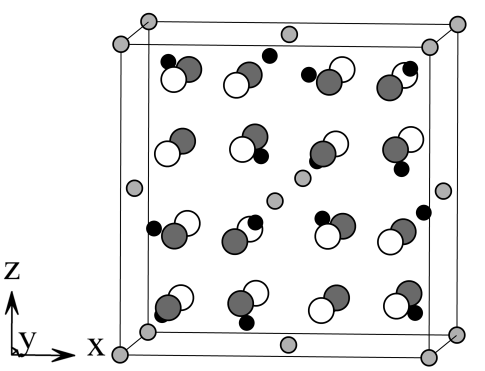

a

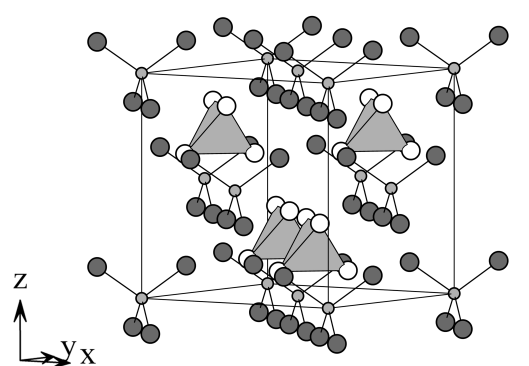

b

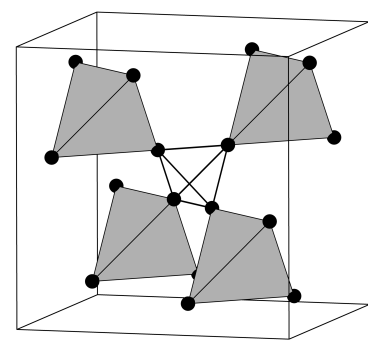

C

FIG. 8. Calculated low-symmetrical anion ordered spinel structure with the space group $F \overline{4} 3 \mathrm{~m}$ and with the structural formula $\mathrm{A}^{4(a)} \mathrm{B}_{4}^{16(e)} \mathrm{X}_{4}^{16(e)} \mathrm{X}_{4}^{\prime 16(e)}$. Atom presentation of ordered spinel structures (a), tetrahedra in ordered spinel structure (b), $\mathrm{B}^{16(e)}$-metallic tetrahedral (nanoclusters) (c). A substance with composition $\operatorname{Re}_{4} \mathrm{As}_{6} \mathrm{~S}_{3}$ has a similar structure. In this structure there are $\mathrm{Re}_{4}$-clusters 
The position of atom $\mathrm{A}$ in the spinel-type (previously the 8a position) splits into two different positions of multiplicity 4 each, one at $(0,0,0)$, surrounded by four atoms from the first sublattice (in $\operatorname{Re}_{4} \mathrm{As}_{6} \mathrm{~S}_{3}, 4$ arsenic atoms), the other at $(3 / 4,3 / 4,3 / 4)$, surrounded by 4 atoms from the second sublattice (in $\operatorname{Re}_{4} \mathrm{As}_{6} \mathrm{~S}_{3} 4 \mathrm{As}_{3} \mathrm{~S}$ atoms) [28]. A central arsenic is found in $(0,0,0)$, producing an $\mathrm{As}_{5}$ clusters. The atom $\mathrm{B}$, in the ideal spinel at the octahedral site $16 \mathrm{~d}$ (at $3 / 8,3 / 8,3 / 8$ ), is moved out of its ideal position, shifting along the body diagonal of the unit cell (coordinates $(x, x, x)$ ). This produces different interatomic distances, allowing contacts with other atoms in symmetry-related $B$ positions [28]. In $\operatorname{Re}_{4} \mathrm{As}_{6} \mathrm{~S}_{3}$, the rhenium atoms have short contacts at a distance of $2.776 \AA[28]$.

The $B_{4}$ and $B_{2} B_{2}^{\prime}$ clusters in lacunar $A B_{4} X_{8}$ spinels. The structural formula of these compounds is $\mathrm{A}^{\prime 4 a} \mathrm{~B}_{4}^{16 e} \mathrm{X}_{4}^{\prime 16 e} \mathrm{X}_{4}^{16 e}$. The filling of half of the A sites with metal atoms gives the $\mathrm{GaMo}_{4} \mathrm{~S}_{8}$ type of structure, which is found for $\mathrm{AB}_{4} \mathrm{X}_{8}(\mathrm{~A}=\mathrm{Ga}, \mathrm{Al}, \mathrm{Ge} ; \mathrm{B}=\mathrm{V}, \mathrm{Nb}, \mathrm{Na}, \mathrm{Mo} ; \mathrm{X}=\mathrm{S}, \mathrm{Se})$ (Fig. 9) and $\mathrm{AMo}_{2} \mathrm{Re}_{2} \mathrm{~S}_{8}(\mathrm{~A}=\mathrm{Zn}$, $\mathrm{Fe}, \mathrm{Co}, \mathrm{Ni})[26,27]$.

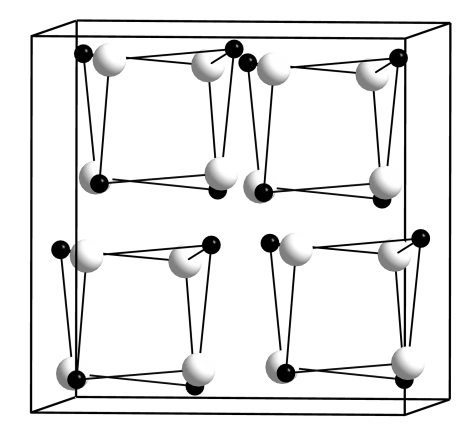

a

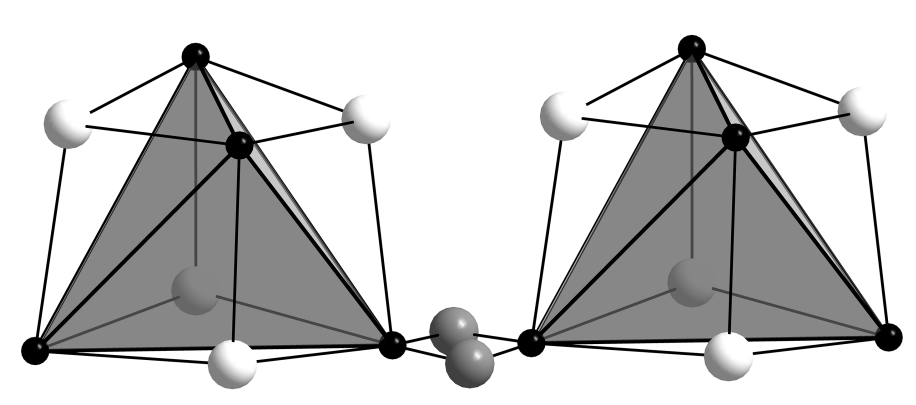

b

FIG. 9. Calculated crystal structure of the $\mathrm{AB}_{4} \mathrm{X}_{8}$ type compounds containing $\mathrm{B}_{4}^{16 e}$ clusters (a), the bridge between two $\mathrm{B}_{4}^{16 e}$ clusters mediated by two $\mathrm{X}^{\prime 16 e}$ atoms (b)

The structure of these a cation-deficient ordered spinels consists of heterocubane-like $\mathrm{B}_{4} \mathrm{X}_{4}^{n+}$ units and $\mathrm{AX}_{4}^{n-}$ tetrahedra arranged in a similar manner to the ions in the rock-salt structure (Fig. 9(a)). The 8a site $(0,0,0)$ of the spinel splits into sites $4 \mathrm{a}(0,0,0)$ and $4 \mathrm{c}(1 / 4,1 / 4,1 / 4)$ in $\mathrm{F} \overline{4} 3 \mathrm{~m}$-phase, where only $4 \mathrm{a}$ is occupied by atoms. The $16 \mathrm{~d}$ site $(5 / 8,5 / 8,5 / 8)$, occupied by the $\mathrm{B}$ atoms, becomes the $16 \mathrm{e}$ site $(x, x, x)$ in $\mathrm{F} \overline{4} 3 \mathrm{~m}$, which allows the $\mathrm{B}$ atoms in this site to dislocate along the body diagonal of the unit cell. Within these clusters the B-B distances are compatible with the formation of metallic bonds, while the large intercluster distances prevent metal-metal bonding. This peculiar topology leads to the formation of molecular-like electronic states within the clusters.

The formation of $\mathrm{B}_{4}$ clusters causes significant changes in the physical properties. The metal electrons not incorporated in $\mathrm{M}-\mathrm{S}$ bonds localize in cluster molecular orbitals. Because the clusters are widely separated, orbitals of different clusters do not overlap and the compound becomes nonmetallic. Electric conduction takes place in case the electrons hop between the clusters. These materials are Mott insulators, in which electrical conduction occurs by the hopping of electrons between clusters separated by relatively large distances $(\approx 4 \AA)$ and not between single atoms as in classical Mott insulators like $\mathrm{NiO}$.

It has been suggested that $\mathrm{GaV}_{4} \mathrm{~S}_{8}$ behaves as an electron glass at low temperatures, while $\mathrm{GaNb}_{4} \mathrm{~S}_{8}$ as recently has been shown to undergo a pressure-induced transition to a superconducting state $(T c \approx 4 \mathrm{~K}$ at $23 \mathrm{GPa}$ ) [34]. The large separation of the tetrahedral metal $\mathrm{B}_{4}$ clusters is believed to be the origin of strong electron correlations (Fig. 9(b)).

Recently, several correlation effects have been reported for $\mathrm{AB}_{4} \mathrm{X}_{8}$ lacunar spinels, including pressure-induced superconductivity [35], bandwidth-controlled metal-to-insulator transition [36,37], large negative magnetoresistance [38], a two-dimensional topological insulating state [39], resistive switching through electric field-induced transition [40-42], emergence of orbitally driven ferroelectricity [43], and an extended Néel-type skyrmion phase [44].

Contrary to the spinel structure, the $\mathrm{B}$ atoms of the cubes shift toward the cube center creating a $\mathrm{B}_{4}$ regular tetrahedral cluster with short metal-metal bond lengths (2.8 to $2.9 \AA)$, and the B atoms of the other type cubes shift toward the cube outside, leading to the metal-metal distances of about $4 \AA$, which is too long to be considered as bonds. 
Ordered spinels of $A_{x} B_{2} X_{4}$ type, where $A=A l, G a, B=M o, V, C r$ and $X=S$, Se. The structural formula ordered spinels in this case is $\mathrm{A}_{2 x} \square_{2(1-x)} \mathrm{B}_{4}^{16 e} \mathrm{X}_{4}^{\prime 16 e} \mathrm{X}_{4}^{16 e}$. The examples of such type ordered spinels are $\mathrm{Ga}_{0.67} \mathrm{Mo}_{2} \mathrm{~S}_{4}$, $\mathrm{Ga}_{0.5} \mathrm{~V}_{2} \mathrm{~S}_{4}, \mathrm{AI}_{0.75} \mathrm{Mo}_{2} \mathrm{~S}_{4}$ and $\mathrm{Ga}_{0.67} \mathrm{Cr}_{2} \mathrm{~S}_{4}$ [23-25]. According to our concept, $\mathrm{B}_{4}$-clusters are also in the structures of these substances.

\section{5. $\mathrm{B}_{4}$-clusters in ordered Laves phases with $\mathrm{MgCu}_{4} \mathrm{Sn}$ structure type}

The crystal structure of Laves phases can be either a cubic C15 $\left(\mathrm{MgCu}_{2}\right)$, hexagonal C14 $\left(\mathrm{MgZn}_{2}\right)$ or dihexagonal $\mathrm{C} 36\left(\mathrm{MgNi}_{2}\right)$. The $\mathrm{C} 15$ phase has more than 1000 binary and ternary forms among the three types of Laves phases. The $\mathrm{MgCu}_{2}$, type structure (Strukturbericht symbol C15) is face-centered cubic, belonging to the space group $\mathrm{Fd} \overline{3} \mathrm{~m}$ with 8 formula units per non-primitive cubic unit cell. The $\mathrm{A}$ sublattice of the $\mathrm{MgCu}_{2} \mathrm{has}$ the structure of diamond net, while the B-sublattice can be described in terms of B-tetrahedra connected by their vertices. So far, more than 80 of such ordered compounds have been reported [45].

The structure formula of ordered Laves phases $\mathrm{C} 15$ may be obtained from structural formula ordered spinel $\mathrm{A}^{\prime 4 a} \mathrm{~A}^{4 c} \mathrm{~B}_{4}^{16 e} \mathrm{X}_{4}^{\prime 16 e} \mathrm{X}_{4}^{16 e}$ if we will consider atoms positions $\mathrm{X}_{4}^{\prime 16 e}$ and $\mathrm{X}_{4}^{16 e}$ as vacant. The structural formula of the ordered Laves phases is $\mathrm{A}^{\prime 4 a} \mathrm{~A}^{4 c} \mathrm{~B}_{4}^{16 e}$. The $\mathrm{A}^{\prime 4 a} \mathrm{~A}^{4 c} \mathrm{~B}_{4}^{16 e}$ structure is presented as an example in Fig. 10. The $\mathrm{B}$-atoms build up the three-dimensional network of slightly distorted corner-sharing $\mathrm{B}_{4}$-tetrahedra. The $\mathrm{A}$ and $\mathrm{A}^{\prime}$ atoms fill larger cages with coordination number 16 (Frank-Kasper polyhedra [46, 47]) within this network. The small distortion of the B-tetrahedra is due to the difference in size between $\mathrm{A}$ and $\mathrm{A}^{\prime}$ atoms [48]. For a more detailed discussion on the crystal chemistry and chemical bonding in such Laves phases we refer to review articles [49-51] and references which present there in.

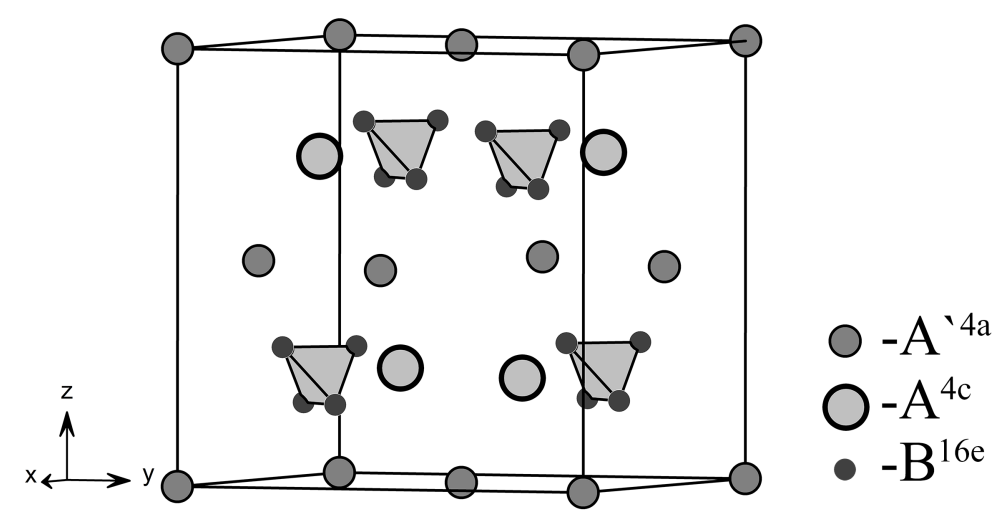

FIG. 10. The crystal structure of $\mathrm{A}^{\prime 4 a} \mathrm{~A}^{4 c} \mathrm{~B}_{4}^{16 e}$ (the structural type of $\mathrm{MgCu}_{4} \mathrm{Sn}$ ). The threedimensional network of corner-sharing $\mathrm{B}_{4}$-tetrahedra is emphasized

\section{6. $\mathbf{A}_{4}$ and $\mathbf{B}_{4}$-clusters in ordered pyrochlore structures}

Compounds $\mathrm{A}_{2} \mathrm{~B}_{2} \mathrm{O}_{6} \mathrm{X}$ with pyrochlore structure are notable for the significant variety of their physical properties: electric (semiconductors, metals, superionics), ferroelectric, magnetic (ferromagnetic), piezoelectric, catalitic. Recently in this family of materials superconductors with structures $\mathrm{Cd}_{2} \mathrm{Re}_{2} \mathrm{O}_{7}(T c=1 \mathrm{~K})$ and $\mathrm{KOs}_{2} \mathrm{O}_{6}$ $(T c=9.6 \mathrm{~K})$ were opened $[52,53]$. Such a wide spectrum of properties is caused substantially by processes of the structural ordering of atoms.

The crystal structure of cubic pyrochlore-type oxides, which have the general formula $\mathrm{A}_{2} \mathrm{~B}_{2} \mathrm{O}_{6} \mathrm{X}$, is composed of a framework of corner shared $\mathrm{BO}_{6}$ octahedra. Here, the $\mathrm{X}$ site is partially or fully occupied by an oxygen atom. The space group of pyrochlore was originally suggested to be $\mathrm{Fd} \overline{3} \mathrm{~m}[54,55]$, in which the $\mathrm{X}$ site is randomly and partially occupied. Later investigation by a powder neutron diffraction experiment [56] showed that the space group is $\mathrm{F} \overline{4} 3 \mathrm{~m}$; in this model vacancies in the $\mathrm{X}$ site are ordered. In $\mathrm{Fd} \overline{3} \mathrm{~m}$-pyrochlore structure $\mathrm{A}_{2}^{16 c} \mathrm{~B}_{2}^{16 d} \mathrm{O}_{6}^{48 f} \mathrm{X}^{8 a}$ atoms occupy four nonequivalent crystallographic positions: cations A and B occupy $16 \mathrm{c}$ and $16 \mathrm{~d}$ Wyckoff positions, and anions occupy 48f and 8a Wyckoff positions. The metal atoms forming clusters, occupy in pyrochlores, as well as in spinel, 16d Wyckoff positions of space group Fd $\overline{3} \mathrm{~m}$. Also, clusters are formed by atoms occupying $16 \mathrm{c}$ Wyckoff positions. The frustrated geometry arises from sublattices of corner-sharing tetrahedra, which are present for both the $\mathrm{A}$ and $\mathrm{B}$ cations. 
We have made the prediction of the existence of a new type of cluster materials on the basis of pyrochlore family with the ordered arrangement of anions $\mathrm{O}$ and $\mathrm{X}$ (type of order 1:1); we have also submitted a calculated structure of pyrochlore (Fig. 11).

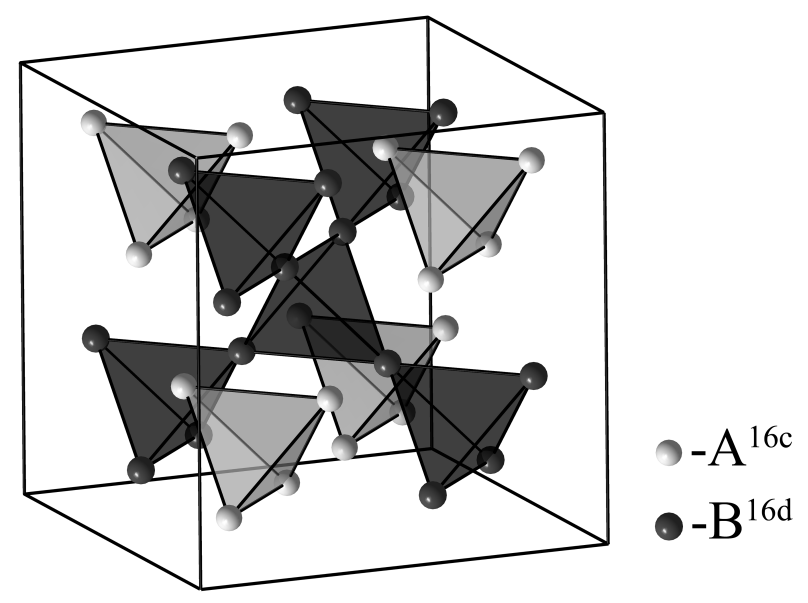

FIG. 11. A calculated elementary cell of pyrochlore, a network of $\mathrm{B}_{4}$ - and $\mathrm{A}_{4}$-tetrahedra. Atoms in $48 \mathrm{f}$ and $8 \mathrm{a}$ Wyckoff positions are not shown

Metal clusters in the structure of ordered pyrochlore are formed due to displacement of cations in $16 \mathrm{~d}$ and $16 \mathrm{c}$ Wyckoff positions. Thus, the theory predicts the existence of four types of metal clusters - compressed and expanded tetrahedra. These tetrahedra form two a three-dimensional network as it is schematically shown on Fig. 11. To explain the unusual physical properties of pyrochlore, it is essential to have direct interactions of metal - metal. These interactions are realized in two networks of $\mathrm{A}_{4}$ - and $\mathrm{B}_{4}$-tetrahedra.

\section{Summary}

Various interesting phenomena concerning the spin, orbital and charge degrees of freedom on these sublattices have been observed in structures with pyrochlore sublattices caused by geometrical frustration. Typical examples are the Verwey transition in $\mathrm{Fe}_{3} \mathrm{O}_{4}$ [57,58], a heavy-fermion state in $\mathrm{LiV}_{2} \mathrm{O}_{4}$ [59], and a heptamer formation in $\mathrm{AlV}_{2} \mathrm{O}_{4}$ [60].

In this work we have established that the structural mechanism of $\mathrm{B}_{4}$-cluster formation in crystals with geometrically frustrated pyrochlore sublattices has universal character. The reason of universal character for $\mathrm{B}_{4}$ cluster formation is caused by the fact of one the same critical irreducible representation $\left(\kappa_{11}\left(\tau_{4}\right)\right)$ which generates the same changes in the geometry of pyroclore sublattices of high-symmetry Fd $\overline{3} \mathrm{~m}$-phases. This conclusion has been illustrated for crystals that belong to different structural types.

We have also showed that cluster formation accompany changes in the geometry of pyrochlore sublattices by appearance of two different B-B-distances. It is so called "breathing" pyroclore sublattice which are in all structural types with low-symmetry F $\overline{4} 3 \mathrm{~m}$ space group. The "breathing" pyrochlore lattice must be an important factor to explore interesting phenomena in frustrated magnets.

We believe that to understand of the origin of magnetic and electrical properties in a given class of materials are important theoretical results as well (hypertetrahedron formation, bridges between tetrahedral metal clusters, "breathing" rings of tetrahedra).

Future tasks will be connected with investigation of the symmetry reduction from space group $\mathrm{Fd} \overline{3} \mathrm{~m}$ to $\mathrm{F} \overline{4} 3 \mathrm{~m}$ in pyrochlore family and the magnesium aluminum-chromium system with composition intermetallic compound $\mathrm{Mg}_{3} \mathrm{Cr}_{2} \mathrm{AI}_{18}$ [61] more detailed.

\section{Acknowledgements}

The reported study was funded by RFBR, according to research project no. 16-32-60025 mol_a_dk (Talanov M. V.). 


\section{References}

[1] Sadoc J.F., Mosseri R. Geometrical Frustration. Cambridge: Cambridge University Press, 2006, 319 p.

[2] Lacroix C., Mendels P., Mila F. Introduction to Frustrated Magnetism. Materials, Experiments, Theory. Springer-Verlag, Berlin, 2011, $128 \mathrm{p}$.

[3] Ramirez A.P. Handbook on Magnetic Materials. Amsterdam: Elsevier Science, 2001, 13, 423 p

[4] Anderson P. Ordering and Antiferromagnetism in Ferrites. Phys. Rev., 1956, 102, P. 1008-1013.

[5] Bernal J.D., Fowler R.H. A Theory of Water and Ionic Solution, with Particular Reference to Hydrogen and Hydroxyl Ions. J. Chem. Phys., 1933, 1, P. 515-548.

[6] Pauling L.J. The Structure and Entropy of Ice and of Other Crystals with Some Randomness of Atomic Arrangement. Am. Chem. Soc., 1935, 57, P. 2680-2684.

[7] Kovalev O.V. Representations of Crystallographic Space Groups. Irreducible Representations, Induced Representations and Corepresentations. London, Taylor and Francis Ltd., 1993, 390 p.

[8] Talanov V.M., Shirokov V.B. Tilting structures in spinels. Acta Cryst. A, 2012, 68, P. 595-606.

[9] Talanov V.M., Shirokov V.B. Atomic order in spinel structure - a group-theoretical analysis. Acta Cryst. A, 2014,70 , P. 49-63.

[10] Talanov V.M., Shirokov V.B., Talanov M.V. Unique atom hyper-kagome order in $\mathrm{Na}_{4} \mathrm{Ir}_{3} \mathrm{O}_{8}$ and in low-symmetry spinel modifications. Acta Cryst. A, 2015, 71, P. 301-318.

[11] Talanov V.M. Anion ordering in spinels. Phys. Stat. sol. (a), 1989, 115, K1-K4.

[12] Talanov V.M. Calculation of Structural Parmeters of Spinels. Phys. Stat. sol. (a), 1981, 106, P. 99-106.

[13] Talanov V.M. Theoretical Grounds of the Natural Classification of Structure Types. Kristallographiya, 1996, 44 (6), P. 979-997; Crystallogr. Rep., 1996, 44, P. 929-946.

[14] Talanov V.M. Structural Mechanism of Cluster Formation in Ordered Chalcogenide Spinels. Phys. Chem. Glass, 2005, 31, P. 323-325.

[15] Talanov M.V., Shirokov V.B., Talanov V.M. Phenomenological thermodynamics and the structure formation mechanism of the CuTi $\mathrm{S}_{4}$ rhombohedral phase. Phys. Chem. Chem. Phys., 2016, 18, P. 10600-10606.

[16] Talanov V.M. Structural mechanism of the ordering of ions in tetrahedral holes in spinels. J. Struct. Chem., 1986, 2, P. 172-176.

[17] Okamoto Y., Nilsen G.J., Attfield J.P., Hiroi Z. Breathing Pyrochlore Lattice Realized in A-Site Ordered Spinel Oxides LiGaCr $4 \mathrm{O}_{8}$ and LiInCr $_{4} \mathrm{O}_{8}$. Phys. Rev. Letters, 2013, 110, 097203(1-5).

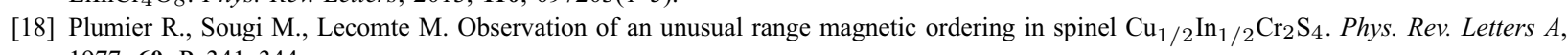
1977, 60, P. 341-344.

[19] Plumier R., Lecomte M., Miedan-Gros A., Sougi M. Observation of a first order macro to microdomain transition in chalcogenide spinel $\mathrm{Ag}_{1 / 2} \mathrm{In}_{1 / 2} \mathrm{Cr}_{2} \mathrm{~S}_{4}$. Physica B, 1977, 86-88, P. 1360-1362.

[20] Nauciel-Bloch M., Plumier R. Magnetic structure in spinel B-site lattice with ordered diamagnetic ions at the A-sites. Solid State Communication, 1971, 9, P. 223-226.

[21] Federov V.E., Mironov Y.V., et al. $\mathrm{Re}_{4} \mathrm{~S}_{4} \mathrm{Te}_{4}$. Acta Crystallogr. C, 1996, 52, P. 1065-1067.

[22] Fedorov V.Y., Mironov Y.V., et al. Cluster Transition Metal Chalcogenide Halides. Russ. Chem. Rev., 2007, 76, P. 529-552.

[23] Brasen D., Vandenberg J.M., et al. Magnetic and Crystallographic Properties of Spinels of the Type $\mathrm{A}_{x} \mathrm{~B}_{2} \mathrm{X}_{4}$ where $\mathrm{A}=\mathrm{Al}$, Ga, and B = Mo, V, Cr. J. Solid State Chemistry, 1975, 13, P. 298-303.

[24] Barz H. New ferromagnetic molybdenum spinels. Mater. Res. Bull., 1973, 8, P. 983-988.

[25] Okuda K., Noguchi S., Asano M., Endo S. Phase transitions of Mo-cluster compounds Ga O.5 $\left(\mathrm{Mo}_{\text {, Re }}\right)_{2} \mathrm{~S}_{4}$. J. Magn. Magnetic Materials, 1990,90 \& 91, P. 148-150.

[26] Ben Yaich H., Jegaden J.C., et al. Nou-veaux chalcogénures et chalcohalogénures à clusters tétraédriques $\mathrm{Nb}_{4}$ ou Ta 4 . J. Less-Common Met., 1984, 102, P. 9-22.

[27] Aminov T.G., Shabunina G.G., Busheva E.V., Novotortsev V.M. Magnetic properties of $\mathrm{Co}_{x}\left(\mathrm{Cu}_{0.5} \mathrm{In}_{0.5}\right)_{1 x} \mathrm{Cr}_{2} \mathrm{~S}_{4}$ solid solutions. Russian Journal of Inorganic Chemistry, 2016, 61 (4), P. 461-469.

[28] Besnard C., Svensson C., Stahl K., Siegrist T. $\operatorname{Re}_{4} \mathrm{As}_{6} \mathrm{~S}_{3}$, a thio-spinel-related cluster system. J. Solid State Chemistry, 2003, 172, P. 446-450.

[29] Fedorov V.E., Mironov Yu.V., et al. Chalcogenide clusters of Group 5- 7 metals. Russian Chemical Reviews, 2007,76, P. 529-552.

[30] Fedorov V.E., Mironov Yu.V., Fedin V.P., Mironov Yu.I. $\operatorname{Re}_{4} \mathrm{~S}_{4} \mathrm{Te}_{4}$. A new mixed rhenium chalcogenide containing a tetrahedral Re 4 cluster. J. Struct. Chem., 1994, 35 (1), P. 146-147.

[31] Schulz Lang E., Abram U., Strahle J. Synthese und Struktur von $\mathrm{Re}_{4}\left(\mu_{3}-\mathrm{Te}\right)_{4}\left(\mathrm{TeBr}_{2}\right)_{4} \mathrm{Br}_{8}$. Z. Anorg. Allg. Chem., 1996, 622, P. $251-253$.

[32] Fedorov V.E., Naumov N.G., et al. Inorganic Coordination Polymers Based on Chalcocyanide Cluster Complexes. J. Struct. Chem., 2002, 43, P. 669-684.

[33] Novotortsev V.M., Kalinnikov V.T., Aminov T.G. Spin glass state in multinary chromium compounds. Russian Journal of Inorganic Chemistry, 2005, 50, P. 54-80.

[34] Pocha R., Johrendt D., Ni B., Abd-Elmeguid M.M. Crystal Structures, Electronic Properties, and Pressure-Induced Superconductivity of the Tetrahedral Cluster Compounds $\mathrm{GaNb}_{4} \mathrm{~S}_{8}, \mathrm{GaNb}_{4} \mathrm{Se}_{8}$, and $\mathrm{GaTa}_{4} \mathrm{Se}_{8}$. J. Am. Chem. Soc., 2005,127 (24), P. 8732-8740.

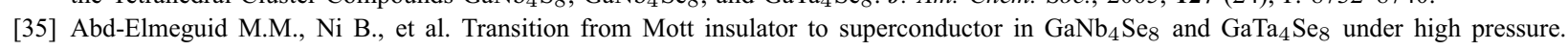
Phys. Rev. Lett., 2004, 93, 126403(1-4).

[36] Ta Phuoc V., Vaju C.,et al. Optical conductivity measurements of $\mathrm{GaTa}_{4} \mathrm{Se}_{8}$ under high pressure: Evidence of a bandwidth-controlled insulator-to-metal Mott transition. Phys. Rev. Lett., 2013, 110, 037401(1-5).

[37] Camjayi A., Acha C., et al. First-order insulator-to-metal Mott transition in the paramagnetic 3D system GaTa 4 Se 8. Phys. Rev. Lett., 2014, 113, 086404(1-5).

[38] Dorolti E., Cario L., et al. Halfmetallic ferromagnetism and large negative magnetoresistance in the new lacunar spinel GaTi ${ }_{3} \mathrm{VS}_{8} . \mathrm{J}_{\text {. }} \mathrm{Am}$. Chem. Soc., 2010, 132, P. 5704-5710.

[39] Kim H.-S., Im J., Han M.J., Jin H. Spin-orbital entangled molecular jeff states in lacunar spinel compounds. Nat. Commun., 2014, 5, P. 3988-3995. 
[40] Dubost V., Cren T., et al. Resistive switching at the nanoscale in the Mott insulator compound GaTa $4 \mathrm{Se}_{8}$. Nano Lett., 2013, 13, P. 36483653.

[41] Stoliar P., Cario L., et al. Universal electric-field-driven resistive transition in narrow-gap Mott insulators. Adv. Mater., 2013, 25, P. 32223226.

[42] Guiot V., Cario L., et al. Avalanche breakdownin $\mathrm{GaTa}_{4} \mathrm{Se}_{8 x} \mathrm{Te}_{x}$ narrow-gap Mott insulators. Nature Commun., 2013, 4, P. 1722-1728,

[43] Singh K., Simon C., et al. Orbital ordering-driven multiferroicity and magnetoelectric coupling in $\mathrm{GeV}_{4} \mathrm{~S}_{8}$. Phys. Rev. Lett., 2014, 113, 137602(1-6).

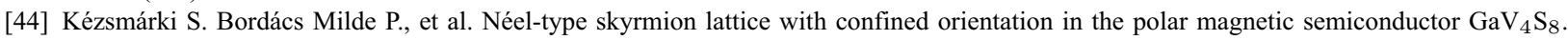
Nat. Mater, 2015, 14, P. 1116-1122.

[45] Linsinger S., Eul M., et al. Structure, homogeneity ranges, magnetic, and electrical properties of the ordered Laves phases ReNi ${ }_{4} \mathrm{Mg}_{3}$ with $\mathrm{MgCu}_{4} \mathrm{Sn}$ type structure. Intermetallics, 2011, 19, P. 1579-1585.

[46] Frank F.C., Kasper J.S. Complex Alloy Structures Regarded as Sphere Packings. I. Definitions and Basic Principles. Acta Crystallogr, 1958, 11, P. 184-190.

[47] Frank F.C., Kasper J.S. Complex alloy structures regarded as sphere packings. II. Analysis and classification of representative structures. Acta Crystallogr., 1959, 12, P. 483-499.

[48] Emsley J. The Elements. Oxford University Press, Oxford, 1989, 256 p.

[49] Nesper R. Bonding patterns in intermetallic compounds. Angew. Chem., 1991, 30, P. $789-817$.

[50] Johnston R.L., Hoffmann R. Structure-Bonding Relationships in the Laves Phases. Z. Anorg. Allg. Chem., 1992 , 616, P. $105-120$.

[51] Nesper R., Miller G.J. A covalent view of chemical bonding in Laves phases $\mathrm{CaLi}_{x} \mathrm{Al}_{2-x}$. J. Alloys Compd., 1993, 197, P. 109-121.

[52] Hanawa M., Muraoka Y., et al. Super-conductivity at $1 \mathrm{~K}$ in $\mathrm{Cd}_{2} \mathrm{Re}_{2} \mathrm{O}_{7}$. Phys. Rev. Lett., 2001, 87, 187001(1-4).

[53] Yamaura J.-I., Yonezawa S., Muraoka Y., Hiroi Z. Crystal structure of the pyrochlore oxide superconductor KOs ${ }_{2} \mathrm{O}_{6}$. Journal of Solid State Chemistry, 2006, 179, P. 336-340.

[54] Shah J.S., Hahn W.C. Material characterization of thick film resistor pastes. IEEE Trans. Compon. Hybrides and Manuf. Technol., 1978, 303 P. 383-385.

[55] Longo J.M., Raccah P.M., Goodenough J.B. $\mathrm{Pb}_{2} \mathrm{M}_{2} \mathrm{O}_{7-x}(\mathrm{M}=\mathrm{Ru}$, Ir, Re) - preparation and properties of oxygen deficient pyrochlores. Mat. Res. Bull., 1969, 4, P. 191-202.

[56] Beyerlein R.A., Horowitz H.S., et al. Neutron diffraction investigation of ordered oxygen vacancies in the defect pyro-chlores, $\mathrm{Pb}_{2} \mathrm{Ru}_{2} \mathrm{O}_{6.5}$ and $\mathrm{PbT}_{1} \mathrm{Nb}_{2} \mathrm{O}_{6.5}$. Solid State Chem., 1984, 51, P. 253-266.

[57] Verwey E.J.W. Electronic Conduction of Magnetite $\left(\mathrm{Fe}_{3} \mathrm{O}_{4}\right)$ and its Transition Point at Low Temperatures. Nature (London), 1939, 144, P. 327-328.

[58] Senn M.S., Wright J.P., Attfield J.P. Charge order and three-site distortions in the Verwey structure of magnetite. Nature (London), 2012, 481, P. 173-176.

[59] Kondo S., Johnston D.C., et al. $\mathrm{LiV}_{2} \mathrm{O}_{4}$ : A Heavy Fermion Transition Metal Oxide. Phys. Rev. Lett., 1997, 78, P. $3729-3732$.

[60] Horibe Y., Shingu M., et al. Spontaneous Formation of Vanadium Molecules in a Geometrically Frustrated Crystal: $\mathrm{AlV}_{2} \mathrm{O}_{4}$. Phys. Rev. Lett., 2006, 96, 086406(1-4).

[61] Samson S. The Crystal Structure of the Intermetalic Compound $\mathrm{Mg}_{3} \mathrm{Cr}_{2} \mathrm{AI}_{18}$. Acta Cryst., 1958, 11, P. 851-857. 